ARTIGO ORIGINAL ORIGINAL ARTICLE

\title{
Análise do custo-efetividade da implantação de uma Unidade de Prática Integrada no cuidado de idosos com fratura do quadril
}

\author{
Cost-effectiveness analysis of the implantation of a \\ Multidisciplinary Unit for elderly with hip fracture
}

Natália Resende Avelino', Alfredo Chaoubah², Guilherme Côrtes Fernandes³, Fabiano Bolpato Loures', Guillermo Patrício Ortega³, Flávia Lícia Rodrigues Magacho', Patrícia Jorge Gonçalves

DOI: $10.21115 / J B E S . v 12 . n 2 . p 121-7$

\section{Palavras-chave:}

custo-efetividade, fraturas do quadril, idoso, hospitalização

\section{Keywords:}

cost-effectiveness, hip fractures, elderly, hospitalization

\section{RESUMO}

Objetivo: Avaliar o custo-efetividade da internação de idosos com fratura do quadril antes e após a implantação de uma unidade multidisciplinar em um serviço de Ortopedia. Métodos: Estudo observacional retrospectivo de caráter quantitativo. Cinquenta e três idosos foram submetidos ao tratamento usual e 219 foram assistidos pela equipe multidisciplinar. Para avaliação dos custos e efetividade, foram utilizados os testes $t$ de Student e qui-quadrado, com nível de significância de 0,05. Para a análise de custo-efetividade, com perspectiva do prestador de saúde, considerando os custos diretos médicos, foi utilizado o modelo estatístico do tipo árvore de decisão. A comparação entre as duas alternativas foi medida pela razão de custo-efetividade incremental. Resultados: Após a introdução da unidade multidisciplinar, o tempo entre o trauma e a cirurgia diminuiu de 4,21 dias para 2,47 ( $p<0,001)$, a permanência, de 8,78 dias para 6,58 $(p=0,041)$ e a mortalidade, de 22,6\% para $8,2 \%(p<0,001)$. O custo do tratamento nos dois grupos não apresentou diferença significativa ( $p=$ 0,838). Conclusão: A introdução da unidade multidisciplinar se mostrou custo-efetiva, uma vez que resultou em menor tempo de internação hospitalar e menor mortalidade intra-hospitalar.

\section{ABSTRACT}

Objective: Evaluating the cost-effectiveness of the hospitalization of elderly with hip fracture before and after the implantation of a multidisciplinary unit in a service of orthopedics. Methods: Quantitative retrospective observational study. Fifty-three elderly were submitted to the usual treatment and 219 were assisted by the multidisciplinary team. The $t$ Student test and the chisquared test were used to assess factors associated with cost variation and effectiveness, with a significance level of 0.05 . For the cost-effectiveness analysis, from the perspective of the health provider considering the direct medical costs, the decision tree statistical model was used. The comparison between the two alternatives was measured by the incremental cost-effectiveness ratio. Results: After introduction of the multidisciplinary unit, the time between trauma and surgery decreased from 4.21 days to $2.47(p<0.001)$, the length of stay from 8.78 days to $6.58(p=0.041)$ and mortality from $22.6 \%$ to $8.2 \%$ ( $p<0.001$ ). The cost of treatment in the two groups did not present a significant difference $(p=0.838)$. Conclusion: The introduction of the multidisciplinary unit was cost-effective, as it resulted in shorter hospital stay and lower in-hospital mortality.

\footnotetext{
Recebido em: 15/05/2020. Aprovado para publicação em: 28/06/2020.

1. Universidade Federal de Juiz de Fora (UFJF), Juiz de Fora, MG, Brasil.

2. Instituto de Ciências Exatas, Departamento de Estatística, Universidade Federal de Juiz de Fora (UFJF), Juiz de Fora, MG, Brasil.

3. Universidade Presidente Antônio Carlos (UNIPAC), Juiz de Fora, MG, Brasil.

Instituição onde o trabalho foi executado: Santa Casa de Misericórdia de Juiz de Fora, MG, Brasil.

Informações sobre auxílios recebidos sob a forma de financiamento, equipamentos ou medicamentos: Não se aplica. Congressos onde o estudo foi apresentado: Não se aplica.

Autor correspondente: Natália Resende Avelino. Praça Menelick de Carvalho, 46/401, Bairro Santa Helena, Juiz de Fora, MG, Brasil.
} CEP: 36015-330. Telefone: (32) 99964-9355. E-mail: nat_avelino@hotmail.com 


\section{Introdução}

A osteoporose é uma das doenças crônico-degenerativas que acometem os idosos e que representa um importante problema de saúde pública, pois aumenta o risco de fraturas, principalmente a de fêmur (Pinto Neto et al., 2002), a qual geralmente necessita de intervenção imediata e, consequentemente, de hospitalização, representando um alto custo social (Fernandes et al., 2011).

Devido à idade, a prevalência de comorbidades é alta. A cirurgia, a reabilitação pós-operatória e a alta hospitalar podem potencialmente ser adiadas se problemas médicos coexistentes não forem apropriadamente administrados, o que pode levar a declínio funcional, cuidados de longo prazo e até óbito (Inouye et al., 1993).

Logo, o desenvolvimento de atendimento multidisciplinar pode contribuir com a redução do encargo médico e social dessa doença (Flikweert et al., 2014).

Em 2006, Michael Porter e Elizabeth Teisberg introduziram o conceito de agenda de valor em seu livro Redefining Health Care. Nos serviços de saúde, o propósito fundamental deve ser o de agregar valor aos pacientes, alcançando meIhores resultados com o menor custo; para isso, o sistema deve estar centrado no paciente, estruturado em torno de suas necessidades.

Para melhorar os resultados e a eficiência para pacientes e agregar valor, os provedores de saúde devem organizar equipes interdisciplinares em torno de condições específicas (Lee, 2010).

Uma Unidade de Prática Integrada (UPI) está organizada em torno de uma doença ou um conjunto de condições relacionadas, sendo o serviço prestado por uma equipe multidisciplinar que assume a responsabilidade pelo ciclo completo de atendimento, incluindo cuidados de reabilitação, atendimento hospitalar e ambulatorial e apoio aos serviços. A implantação dessas unidades gera tratamento mais rápido, melhores resultados e custos mais baixos (Porter \& Lee, 2013).

Por ser a fratura do quadril um problema de saúde pública, pesquisas na área podem impactar no prognóstico dessa doença, reduzindo suas complicações e aumentando a expectativa e a qualidade de vida dos pacientes.

O presente estudo teve como objetivo desenvolver uma análise econômica em saúde do tipo custo-efetividade. Os desfechos foram as razões de custo/efetividade para o tempo de internação e mortalidade entre os pacientes idosos com fratura de quadril tratados antes e após a implantação da UPI no setor de Ortopedia.

\section{Métodos}

A avaliação econômica da implantação da unidade multidisciplinar considerou o método CHEERS (Consolidated Health Economic Evaluation Reporting Standards) - padrões consolidados para o relato de avaliações econômicas em saúde.
Foi realizada uma análise econômica em saúde, do tipo razão de custo-efetividade, com a perspectiva do prestador de saúde, considerando os custos diretos médicos na moeda Real (R\$). O horizonte temporal foi de curto prazo. Foi criado um modelo de análise do tipo árvore de decisão, devido ao fato de o procedimento estudado ser de curta duração e pouco complexo.

Tratou-se de um estudo observacional do tipo coorte não concorrente, com coleta de dados retrospectiva e criação de amostra sistemática. Essa amostra partiu da análise dos prontuários eletrônicos de todos os pacientes idosos submetidos à cirurgia ortopédica na Santa Casa de Misericórdia de Juiz de Fora/MG, no período de 10 de janeiro de 2010 a 31 de dezembro de 2016, com exceção do ano de 2012, quando se deu a implantação da UPI no serviço de Ortopedia da referida instituição. A exclusão do ano de 2012 teve como intuito diminuir possíveis vieses referentes ao período imediatamente antes e imediatamente após a implantação da UPI no serviço, por isso as atividades ainda não haviam sido completamente implementadas.

A pesquisa foi submetida ao Comitê de Ética em Pesquisa da Santa Casa de Misericórdia de Juiz de Fora/MG, sendo aprovada conforme o Parecer Consubstanciado no 1.965.150, de 14/03/2017.

Antes da implantação da unidade multidisciplinar, a fratura de fêmur era conduzida pelo cirurgião e, quando necessário, outros profissionais eram demandados. A UPI de Ortopedia foi implementada em setembro/2012 e possui 16 leitos, atuando na média e alta complexidade. $O$ paciente é assistido integralmente pela equipe multidisciplinar, que se reúne periodicamente e é formada por profissionais médicos, enfermeiros, técnicos de enfermagem, nutricionistas, fonoaudiólogos, psicólogos, assistentes sociais, farmacêuticos, fisioterapeutas e auxiliares administrativos, todos como foco na segurança do paciente, humanização, melhores resultados e redução de custos.

A busca no prontuário eletrônico da instituição pesquisada teve início a partir do Código Internacional de Doenças (CID) em sua décima revisão, o CID-10. Selecionamos os pacientes que apresentaram como diagnóstico de internação a fratura do colo do fêmur (S72.0) e a fratura pertrocantérica (S72.1).

Os critérios de inclusão foram idade igual ou acima de 60 anos, diagnóstico compatível (CID-10: 572.0 e S72.1), procedimento cirúrgico realizado e pacientes internados pelo Sistema Único de Saúde (SUS).

Os critérios de exclusão foram trauma de alta energia, doença óssea secundária, exceto osteoporose, situação clínica de base gravíssima impeditiva do tratamento cirúrgico e tratamento conservador.

Foram coletadas também as seguintes variáveis: sexo, idade e tipo de fratura, data de admissão no hospital do estudo, da cirurgia e da alta/óbito, a fim de conhecer o tempo até a cirurgia e o tempo de permanência. 
Os custos diretos foram levantados no centro de custos do hospital, em números totais e por categorias para cada paciente: internação em enfermaria; internação em centro de terapia intensiva (CTI); honorário médico; procedimento (exames + sala cirúrgica); material médico e medicamentos.

Os custos foram ajustados pelo índice da Fundação Instituto de Pesquisas Econômicas (Fipe), na ponderação sobre os preços em saúde (Fipe Saúde) e corrigidos para janeiro de 2018.

Os custos e os desfechos foram controlados pelas variáveis independentes: sexo, idade, tipo de fratura e tempo entre o trauma e a cirurgia.

A amostra foi, então, dividida em dois grandes grupos: idosos com fratura do quadril tratados cirurgicamente antes da implantação da UPI (anos 2010 e 2011) e após a implantação da referida unidade multidisciplinar (anos 2013 a 2016).

Os grupos foram inicialmente comparados pelo teste $t$ de Student quanto às médias para idade e tempo entre o trauma e a cirurgia, como variável contínua, e pelo qui-quadrado quanto ao sexo e tipo de fratura, como variáveis dicotômicas.

Os dados foram representados por média e desvio-padrão (DP) ou frequência relativa e absoluta. Para a execução da análise estatística, foi utilizado o software Statistical Package for Social Sciences (SPSS) v.21. O nível de significância considerado foi 0,05.

Para a análise de custo-efetividade da implantação da UPI de Ortopedia, foi utilizado o modelo estatístico do tipo árvore de decisão. O modelo desenvolvido no software TreeAge Suite 2011 estimou a efetividade em termos de tempo de internação e mortalidade e os custos associados ao modelo de tratamento (pré-UPI x pós-UPI) na perspectiva do prestador de saúde.

A comparação entre as duas alternativas foi medida pela razão de custo-efetividade incremental (RCEI). O primeiro desfecho de interesse estudado foi a mortalidade, sendo a RCEI = (Custos do tratamento na UPI - Custos do tratamento do modelo tradicional) / (Diferença entre a taxa de sobrevida nos dois grupos). O segundo desfecho foi o tempo de permanência hospitalar e, dessa forma, sua razão de custo-efetividade foi calculada por meio da fórmula RCEl = (Custos do tratamento na UPI - Custos do tratamento do modelo tradicional) / (Diferença entre o tempo de internação nos dois grupos).

\section{Resultados}

A amostra foi composta por 272 pacientes idosos com fraturas do quadril, dos quais 53 foram tratados cirurgicamente antes da implantação da UPI (anos 2010 e 2011) e 219 após a instituição da referida unidade multidisciplinar (anos 2013 a 2016).

Na tabela 1 constam as características sociodemográficas e clínicas dos participantes, além do controle das características das amostras com relação às variáveis independentes.

Uma vez que não foram encontradas diferenças estatisticamente significativas entre o grupo pré-implantação e o pós-implantação da UPI em relação ao sexo, idade e tipo de fratura, com exceção da variável "tempo até cirurgia", a comparação entre eles se fez possível. O tempo de espera para a realização da cirurgia após a implantação da UPI foi menor $(p<0,001)$, demonstrando que o trabalho da equipe multidisciplinar diminui o tempo decorrido entre a fratura e a realização da cirurgia em idosos. Vale ressaltar ainda que, durante o período do referido estudo, não houve mudanças nas técnicas e equipamentos utilizados para o tratamento cirúrgico da fratura do quadril em idosos.

O tempo total de permanência hospitalar foi de 8,78 dias (DP: 5,08) no período pré-implantação e de 6,58 dias (DP: 7,35) no pós-implantação da UPI ( $p=0,041)$.

Dos 53 pacientes com fratura do quadril tratados cirurgicamente antes da implantação da UPI, 12 foram a óbito intra-hospitalar, o que corresponde a 22,6\% da amostra. Após a introdução da referida unidade, essa proporção foi igual a $8,2 \%(p<0,001)$.

Tabela 1. Características sociodemográficas e clínicas da amostra e controle das características das amostras

\begin{tabular}{|c|c|c|c|}
\hline Variável & Pré-UPI & Pós-UPI & $\mathbf{p}$ \\
\hline Sexo & $73,6 \%$ 우 & $67,1 \%$ 우 & 0,496 \\
\hline Idade (anos) & 78,51 (DP: 8,68) & 79,83 (DP: 9,39) & 0,352 \\
\hline Faixa etária & n (\%) & n (\%) & 0,506 \\
\hline $\begin{array}{l}<70 \text { anos } \\
70 \text { a } 80 \text { anos } \\
80 \text { a } 90 \text { anos } \\
\geq 90 \text { anos }\end{array}$ & $\begin{array}{c}12(22,6) \\
14(26,4) \\
24(45,3) \\
3(5,7)\end{array}$ & $\begin{array}{l}41(18,7) \\
62(28,3) \\
89(40,6) \\
27(12,3)\end{array}$ & \\
\hline $\begin{array}{l}\text { Tipo de fratura } \\
\text { Fratura do colo do fêmur } \\
\text { Fratura pertrocantérica }\end{array}$ & $\begin{array}{l}61,5 \% \\
38,5 \%\end{array}$ & $\begin{array}{l}60,3 \% \\
39,7 \%\end{array}$ & 0,782 \\
\hline Tempo até a cirurgia (dias) & 4,21 (DP: 2,94) & $2,47$ (DP: 2,48$)$ & $<0,001$ \\
\hline
\end{tabular}

DP: desvio-padrão; p: probabilidade de significância; UPI: Unidade de Prática Integrada. 
Em relação à necessidade de cuidados intensivos, 13 (24,5\%) pacientes operados nos anos 2010 e 2011 utilizaram o CTI. Já no período após a introdução da unidade multidisciplinar, 18 (8,2\%) necessitaram de terapia intensiva. Observou-se que, dos 13 pacientes operados nos anos 2010 e 2011 que necessitaram de cuidados intensivos, 8 foram a óbito $(61,5 \%)$ e, no período após a introdução da unidade multidisciplinar, dos 18 pacientes encaminhados ao CTI, 11 faleceram (61,1\%).

A média dos custos diretos por categoria e totais se encontra nos gráficos (Figuras 1 e 2).

Com relação à análise econômica, observou-se que, tanto em termos de mortalidade quanto de tempo de internação, a implantação da UPI foi mais custo-efetiva (Figuras 3 e 4).

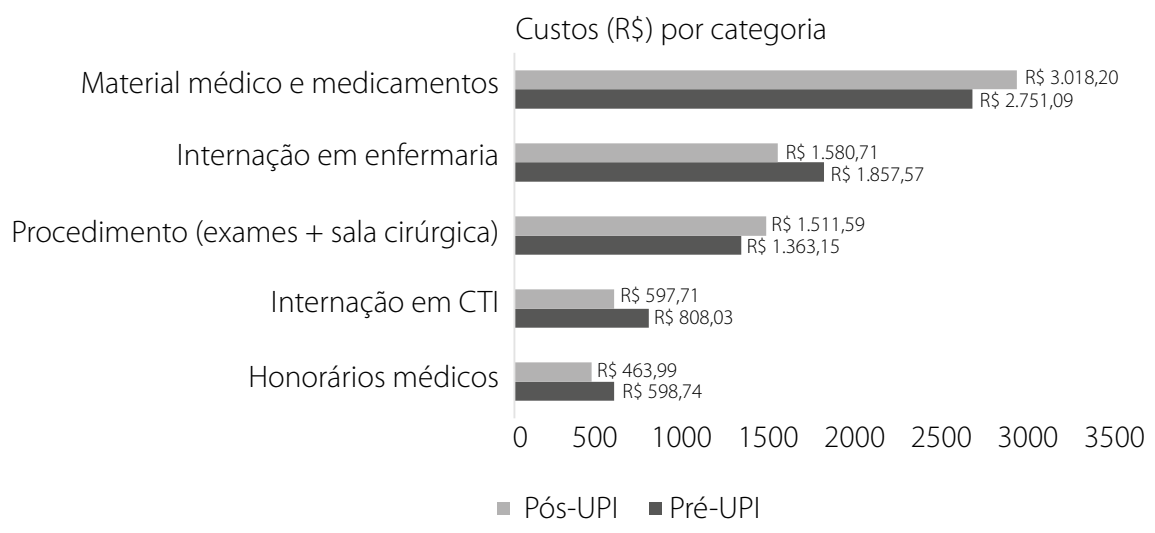

CTI: centro de terapia intensiva; R\$: Real; UPI: Unidade de Prática Integrada.

Figura 1. Média dos custos diretos por categoria.

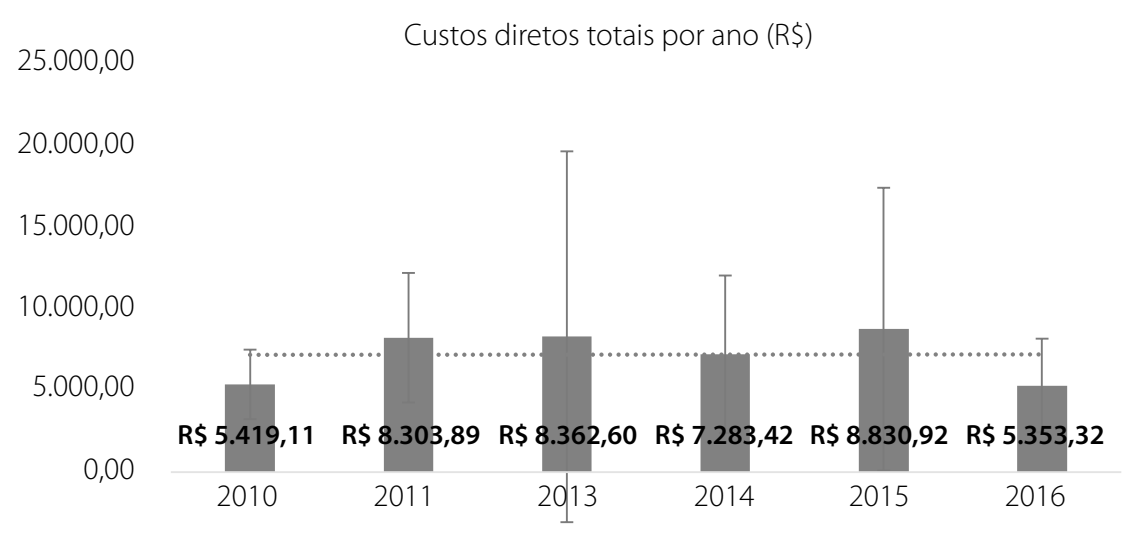

RS: Real.

Figura 2. Média dos custos diretos totais (R\$) por ano.

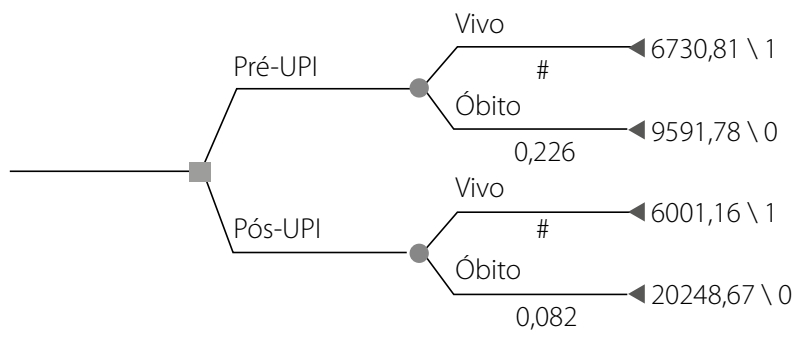

UPI: Unidade de Prática Integrada.

Nota: Valores apresentados em reais (R\$).

Figura 3. Árvore de decisão - Custo-efetividade (mortalidade).

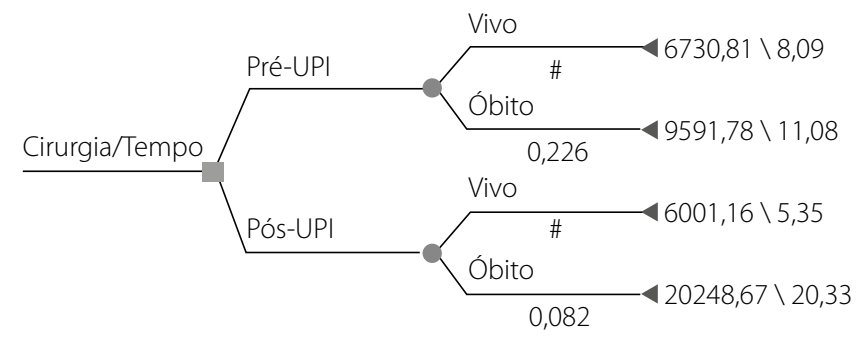

UPI: Unidade de Prática Integrada.

Nota: Valores apresentados em reais (R\$).

Figura 4. Árvore de decisão - Custo-efetividade (tempo de internação). 
A introdução da unidade multidisciplinar se mostrou dominante, não sendo necessário o cálculo da RCEl, uma vez que o custo total da internação pré e pós-UPI não evidenciou diferença significativa $(p=0,838)$, porém a mortalidade e o tempo de internação foram menores após a introdução da UPI (Tabelas 2 e 3).

Tabela 2. Análise de custo-efetividade (mortalidade)

\begin{tabular}{lcc}
\hline Estratégia & Pré-UPI & Pós-UPI \\
\hline Custo & $R \$ 7378,58$ & $R \$ 7172,20$ \\
\hline Custo incremental & & $R \$ 206,38$ \\
\hline Efetividade & 0,774 & 0,918 \\
\hline Efetividade incremental & & $-0,144$ \\
\hline RCEI & & (Dominante) \\
\hline
\end{tabular}

RCEl: razão de custo-efetividade incremental; R\$̦: Real; UPI: Unidade de Prática Integrada.

Tabela 3. Análise de custo-efetividade (tempo de internação)

\begin{tabular}{lcc}
\hline Estratégia & Pré-UPI & Pós-UPI \\
\hline Custo & $R \$ 7378,58$ & $R \$ 7172,20$ \\
\hline Custo incremental & & $R \$ 206,38$ \\
\hline Efetividade & 8,78 & 6,58 \\
\hline Efetividade incremental & & 2,2 \\
\hline RCEI & & (Dominante) \\
\hline
\end{tabular}

RCEl: razão de custo-efetividade incremental; R\$̦: Real; UPI: Unidade de Prática Integrada.

\section{Discussão}

O presente estudo demonstrou uma maior prevalência do sexo feminino em relação ao masculino na ocorrência da fratura de fêmur nos dois grupos comparativos, tal resultado também foi encontrado em estudos publicados por Cooper et al. (2011) e Soares et al. (2014).

Para Arndt et al. (2011) e Edelmuth et al. (2018), o predomínio desse tipo de fratura em mulheres justifica-se pela diminuição da densidade mineral óssea se iniciar antecipadamente no sexo feminino após a menopausa, somado ao fato de as mulheres atingirem o pico de potência muscular antes do sexo masculino e, assim, sofrerem seu declínio primeiramente.

A idade média dos pacientes do estudo ficou entre a sétima e a oitava décadas de vida, semelhante à encontrada nos estudos de Hungria Neto et al. (2011), Belmont Jr. et al. (2014) e Guerra et al. (2017). A fratura do quadril ocorre em idosos com idade mais avançada devido às alterações musculoesqueléticas que predispõem às quedas em razão do comprometimento do desempenho de habilidades motoras (Farias et al., 2017).

Em nosso estudo, nos dois grupos comparados, o tipo de fratura mais prevalente foi a do colo do fêmur, como também foi demonstrado por Quevedo-Tejero et al. (2011), Folbert et al. (2017) e Cheung et al. (2018).

A despeito do tempo entre o trauma e a realização do procedimento cirúrgico, observamos que, antes da implantação da UPI, o tempo médio foi de 4,21 dias (DP: 2,94) e que, após a implantação dela, esse intervalo caiu para 2,47 dias (DP: 2,48$)(p<0,001)$. Assim como em nosso estudo, Farias et al. (2017) evidenciaram que a introdução de cuidados multidisciplinares diminuiu o tempo decorrido entre a fratura e a realização da cirurgia em idosos, de nove para três dias em média.

Alguns autores relatam diminuição na mortalidade quando da realização da cirurgia em 24 horas como Simunovic et al. (2010) e em 48 horas conforme evidenciado por Carretta et al. (2011), enquanto também observamos relatos que não demonstraram benefício na diminuição da mortalidade na cirurgia precoce (Khan et al., 2009). No presente estudo, revelamos que os pacientes tratados após a implantação da UPI que não faleceram aguardaram em média 2,21 dias (DP: $2,11)$ para a realização do procedimento, ao passo que os pacientes que foram a óbito apresentaram tempo médio até a cirurgia de 5,33 dias (DP: 4,12). Deve-se considerar ainda que o tempo entre o trauma e a realização da cirurgia nos pacientes que foram a óbito pode ter sido maior devido à necessidade de tempo de estabilização pré-cirúrgica mais prolongado pela possível existência de mais comorbidades ou maior descompensação delas.

O tempo de internação se mostrou significativo na presente análise quando comparamos os grupos antes da UPI e após a introdução dela ( $p=0,041)$, assim como evidenciado por Leung et al. (2018) e Wallace et al. (2019). Também observamos que, em relação à mortalidade nos dois grupos, os pacientes que foram tratados antes da unidade multidisciplinar e que foram a óbito ficaram em média 11,08 dias internados (DP: 7,65), enquanto os que permaneceram vivos ficaram 8,10 dias no hospital (DP: 3,92). Da mesma forma, os pacientes tratados após a UPI que faleceram permaneceram 20,33 dias no hospital (DP: 18,96) e os que ficaram vivos, 5,35 dias (DP: 3,14).

Segundo Ricci et al. (2015), o tempo de internação hospitalar tem sido identificado como um importante direcionador do custo hospitalar e da utilização de recursos em pacientes com fratura do quadril. O fator mais comumente citado associado ao tempo de internação é a existência de comorbidades nos pacientes internados com fratura de fêmur. Um período de internação mais prolongado é fator agravante para a ocorrência de complicações e pode interferir na taxa de morbimortalidade (Mesquita et al., 2009).

Uma vez que a permanência hospitalar é o componente mais oneroso em relação aos cuidados necessários à fratura de fêmur e, como exposto anteriormente, sofre grande influência quando da existência de comorbidades prévias e complicações, o que pode, inclusive, aumentar a mortalidade, a gestão dos pacientes por meio de modelos de 
coatendimento e, para além disso, os cuidados anteriores à ocorrência da fratura podem gerar uma economia significativa de recursos hospitalares e agregar valor para o paciente (Gupta, 2014).

A taxa geral de mortalidade intra-hospitalar foi de $11 \%$. Em comparação com estudos internacionais como na Austrália, onde a taxa foi de 7,4\% (Zeltzer et al., 2014), e na Espanha, com 5,5\% (Padrón-Monedero et al., 2017), o número de óbitos em nossa amostra foi alto. Já em âmbito nacional, nossos achados são comparáveis com os encontrados por Edelmuth et al. (2018), que observou uma taxa de mortalidade de 11,9\%. No presente estudo, após a implantação da UPI, a mortalidade reduziu de 22,6 para 8,2\%, corroborando os achados de Suarez et al. (2017), que observou diminuição de $23 \%$ para 12,7\%, evidenciando que a participação da equipe multidisciplinar nos cuidados de idosos com fratura de fêmur reduz, entre outros, a taxa de mortalidade.

Os pacientes atendidos anteriormente à introdução dos cuidados multidisciplinares utilizaram mais a unidade de terapia intensiva; o mesmo resultado foi encontrado por Farias et al. (2017). A mortalidade dos pacientes que necessitaram de assistência intensiva foi igual nos dois grupos, de aproximadamente $61 \%$.

Na análise dos custos, não houve diferença significativa entre os dois grupos, com exceção dos gastos relacionados aos honorários médicos. O tratamento dos pacientes antes da implantação da UPI custou, em média, R\$ 7.378,58 e o do grupo assistido pela equipe multidisciplinar ficou em R\$ 7.172,20 em média. Nos dois grupos, os custos relacionados aos materiais médicos/medicamentos e à internação em enfermaria foram os maiores, assim como evidenciado no estudo de Farias et al. (2016).

Apesar de não haver diferença estatisticamente significativa em relação ao custo total de tratamento nos dois grupos ( $p=0,838$ ), a implantação da UPI no cuidado dos pacientes idosos com fratura de quadril mostrou-se mais custo-efetiva quando comparada ao modelo anterior.

Resultados semelhantes foram encontrados por Ginsberg et al. (2013), Lau et al. (2017) e Leal et al. (2017) na avaliação do custo-efetividade da introdução de cuidados multidisciplinares no tratamento de idosos com fratura de quadril.

Como muito bem colocado por Farias et al. (2016), quando da comparação com estudos internacionais, faz-se necessário ponderar as distintas abordagens e organização do tratamento. Na maior parte deles, a análise dos custos considera também a reabilitação geriátrica, o que não ocorre em nosso país, uma vez que ela se dá no âmbito ambulatorial. Também é necessário considerar que a maioria dos estudos internacionais está relacionada à introdução dos cuidados ortogeriátricos, nos quais a abordagem do paciente idoso com fratura de quadril conta com geriatras, médicos-cirurgiões e enfermeiros especialistas. Tal equipe não coincide com a que forma nossa UPI, uma vez que ela é composta por profissionais médicos, enfermeiros, técnicos de enfermagem, nutricionistas, fonoaudiólogos, psicólogos, assistentes sociais, farmacêuticos, fisioterapeutas e auxiliares administrativos, porém ainda não conta com a presença do geriatra.

De acordo com Giusti e Kammerlander (2018), uma série de características distingue os modelos inovadores dos cuidados tradicionais, como a abordagem baseada na avaliação geriátrica abrangente, equipe multidisciplinar capacitada de profissionais de saúde e a configuração e a organização dos cuidados. No entanto, alguns autores defendem que a abordagem do paciente por uma equipe multidisciplinar é o aspecto mais característico de todos os exemplos eficazes e é o único que pode enfrentar com sucesso as questões complexas que envolvem idosos com fratura do quadril.

A implantação da UPI no setor de Ortopedia do Hospital Santa Casa de Misericórdia de Juiz de Fora/MG, além do trabalho multidisciplinar, conta com a realização periódica de reuniões entre a equipe, nas quais a assistência prestada ao paciente é discutida e avaliada, resultando em estatísticas que proporcionam ajustes no processo de gerenciamento, promovendo melhores resultados para o paciente e a instituição.

Por tratar-se de um estudo observacional, baseado em registros assistenciais e administrativos, certas associações podem não afirmar causalidade. A principal limitação desse estudo relaciona-se à impossibilidade da coleta de dados referentes às comorbidades e ao risco cardiológico dos pacientes, em virtude da ausência dessas informações no prontuário, podendo-se subestimar a verdadeira condição clínica dos pacientes da amostra.

Além disso, a comparação não contemporânea impõe algumas limitações, pois não é possível assegurar que as diferenças em efetividade sejam exclusivamente pela implantação da UPI. A impossibilidade de avaliação das comorbidades e a não consideração dos custos intangíveis podem estar relacionadas à melhor efetividade ou, até mesmo, à similaridade dos custos entre as duas alternativas de saúde.

As limitações apresentadas estimulam pesquisas futuras que considerem, por exemplo, as comorbidades prévias, o risco cirúrgico e a inclusão do profissional geriatra na equipe multiprofissional.

\section{Conclusões}

Este estudo demonstrou que a introdução da UPI no tratamento de idosos com fratura de fêmur se mostrou dominante em relação ao período pré-UPI, sendo, assim, mais custo-efetiva. A implantação da UPI resultou em menor tempo para a realização da cirurgia, menor tempo de internação hospitalar e menor mortalidade intra-hospitalar. 


\section{Referências bibliográficas}

Arndt AB, Telles JL, Kowalski SC. O custo direto da fratura de fêmur por quedas em pessoas idosas: análise no setor privado de saúde na cidade de Brasília, 2009. Rev Bras Geriatr Gerontol. 2011;14(2):221-31.

Belmont PJ Jr, Garcia EJ, Romano D, Bader JO, Nelson KJ, Schoenfeld AJ. Risk factors for complications and in-hospital mortality following hip fractures: a study using the National Trauma Data Bank. Arch Orthop Trauma Surg. 2014;134(5):597-604.

Carretta E, Bochicchio V, Rucci P, Fabbri G, Laus M, Fantini MP. Hip fracture: effectiveness of early surgery to prevent 30-day mortality. Int Orthop. 2011;35(3):419-24.

Cheung WH, Shen WY, Dai DL, Lee KB, Zhu TY, Wong RM, et al. Evaluation of a multidisciplinary rehabilitation programme for elderly patients with hip fracture: a prospective cohort study. J Rehabil Med. 2018;50(3):285-91.

Cooper C, Cole ZA, Holroyd CR, Earl SC, Harvey NC, Dennison EM, et al.; IOF CSA Working Group on Fracture Epidemiology. Secular trends in the incidence of hip and other osteoporotic fractures. Osteoporos Int. 2011;22(5):1277-88.

Edelmuth SV, Sorio GN, Sprovieri FA, Gali JC, Peron SF. Comorbidades, intercorrências clínicas e fatores associados à mortalidade em pacientes idosos internados por fratura de quadril. Rev Bras Ortop. 2018;53(5):1-9.

Farias FI, Terra NL, Brum RL, Alves F, Frare CS, Guerra MTE. Fatores determinantes dos custos dos tratamentos para idosos com fratura de quadril. Geriatr Gerontol Aging. 2016;10(4):1-7.

Farias Fl, Terra NL, Guerra MT. Avaliação da efetividade de um programa de atenção ao idoso com fratura de quadril: uma estratégia de rede. Rev Bras Geriatr Gerontol. 2017;20(5):705-16.

Fernandes RA, Araujo DA, Takemoto ML, Sauberman MV. Fraturas do fêmur proximal no idoso: estudo de custo da doença sob a perspectiva de um hospital público no Rio de Janeiro, Brasil. Physis. 2011;21(2):395-416.

Flikweert ER, Izaks GJ, Knobben BA, Stevens M, Wendt K. The development of a comprehensive multidisciplinary care pathway for patients with a hip fracture: design and results of a clinical trial. BMC Musculoskelet Disord. 2014;15:188.

Folbert EC, Hegeman JH, Gierveld R, van Netten JJ, Velde DV, Ten Duis HJ, et al. Complications during hospitalization and risk factors in elderly patients with hip fracture following integrated orthogeriatric treatment. Arch Orthop Trauma Surg. 2017;137(4):507-15.

Ginsberg G, Adunsky A, Rasooly I. A cost-utility analysis of a comprehensive orthogeriatric care for hip fracture patients, compared with standard of care treatment. Hip Int. 2013;23(6):570-5.

Giusti A, Kammerlander C. Comprehensive Geriatric Assessment in Orthogeriatrics. In: Pilotto A, Martin F. Comprehensive Geriatric Assessment. Practical Issues in Geriatrics. Berlin: Springer; 2018. cap. 11, p. 123-32.

Guerra MT, Viana RD, Feil L, Feron ET, Maboni J, Vargas ASG. Mortalidade em um ano de pacientes idosos com fratura do quadril tratados cirurgicamente num hospital do Sul do Brasil. Rev Bras Ortop. 2017:52(1):17-23.

Gupta A. The effectiveness of geriatrician-led comprehensive hip fracture collaborative care in a new acute hip unit based in a general hospital setting in the UK. J R Coll Physicians Edinb. 2014;44(1):20-6.

Inouye SK, Viscoli CM, Horwitz RI, Hurst LD, Tinetti ME. A predictive model for delirium in hospitalized elderly medical patients based on admission characteristics. Ann Intern Med. 1993;119(6):474-81.

Khan SK, Kalra S, Khanna A, Thiruvengada MM, Parker MJ. Timing of surgery for hip fractures: A systematic review of 52 published studies involving 291,413 patients. Injury. 2009;40(7):692-7.
Lau TW, Fang C, Leung F. The effectiveness of a multidisciplinary hip fracture care model in improving the clinical outcome and the average cost of manpower. Osteoporos Int. 2017;28(3):791-8.

Leal J, Gray AM, Hawley S, Prieto-Alhambra D, Delmestri A, Arden NK, et al.; the REFReSH Study Group. Cost-Effectiveness of Orthogeriatric and Fracture Liaison Service Models of Care for Hip Fracture Patients: A Population-Based Study. J Bone Miner Res. 2017;32(2):203-11.

Lee TH. Putting the Value Framework to Work. N Engl J Med. 2010;363(26):2481-3.

Leung FK, Lau TW, Yuen GW, Chan EM, Chan P, Lam RY. Effectiveness of a multidisciplinary approach to geriatric hip fractures in improving clinical outcomes and cost of care. Hong Kong Med J. 2018;24 Suppl 2(1):45-7.

Mesquita GV, Lima MA, Santos AMR, Alves ELM, Brito JN, Martins MCC. Morbimortalidade em idosos por fratura proximal do fêmur. Texto Contexto Enferm. 2009:18(1):67-73.

Hungria Neto JS, Dias CR, Almeida JDB. Características epidemiológicas e causas da fratura do terço proximal do fêmur em idosos. Rev Bras Ortop. 2011;46(6):660-7.

Padrón-Monedero A, López-Cuadrado T, Galán I, Martínez-Sánchez EV, Martin P, Fernández-Cuenca R. Effect of comorbidities on the association between age and hospital mortality after fall-related hip fracture in elderly patients. Osteoporos Int. 2017;28(5):1559-68.

Pinto Neto AM, Soares A, Urbanetz AA, Souza ACA, Ferrari AEM, Amaral B, et al. Consenso brasileiro de osteoporose 2002. Rev Bras Reumatol. 2002:42(6):343-54.

Porter ME, Lee T. A estratégia que irá corrigir os serviços de saúde. Harvard Business Review. 2013:32-49.

Porter ME, Teisberg E. O. Redefining Health Care: Creating Value-based Competitions on Results. Massachusetts: Harvard Business School Press; 2006.

Quevedo-Tejero EC, Zavala-González MA, Hernández-Gamas AC, HernándezOrtega HM. Fractura de cadera en adultos mayores: prevalencia y costos en dos hospitales. Tabasco, Mexico, 2009. Rev Peru Med Exp Salud Publica. 2011;28(3):440-5.

Ricci WM, Brandt A, McAndrew C, Gardner MJ. Factors affecting delay to surgery and length of stay for patients with hip fracture. J Orthop Trauma. 2015;29(3):e109-14.

Simunovic N, Devereaux PJ, Sprague S, Guyatt GH, Schemitsch E, Debeer J, et al. Effect of early surgery after hip fracture on mortality and complications: systematic review and meta-analysis. CMAJ. 2010;182(15):1609-16.

Soares DS, Mello LM, Silva AS, Martinez EZ, Nunes AA. Fraturas de fêmur em idosos no Brasil: análise espaço-temporal de 2008 a 2012. Cad Saúde Pública. 2014;30(12):2669-78.

Suarez S, Pesantez RF, Diaz ME, Sanchez D, Tristancho LJ, Vanegas MV, et al. Impact on Hip Fracture Mortality After the Establishment of an Orthogeriatric Care Program in a Colombian Hospital. J Aging Health. 2017;29(3):474-88.

Wallace R, Angus LDG, Munnangi S, Shukry S, DiGiacomo JC, Ruotolo C. Improved outcomes following implementation of a multidisciplinary care pathway for elderly hip fractures. Aging Clin Exp Res. 2019;31(2):273-8.

Zeltzer J, Mitchell RJ, Toson B, Harris IA, Ahmad L, Close J. Orthogeriatric services associated with lower 30-day mortality for older patients who undergo surgery for hip fracture. Med J Aust. 2014;201(7):409-11. 